\title{
Long Term High-Fat Diet-Induced Modification of Vascular Wall and Perivascular Adipose Tissue-Mediated Oxidative Stress: Con- sequences for Endothelium-Independent Vascular Function in Rats
}

\author{
Caroline Sanchez ${ }^{1,2}$, Vincent Achard ${ }^{1}$, Michel Grino ${ }^{3}$ and Stephane Tanguy ${ }^{1 *}$ \\ ${ }^{1}$ Université Grenoble Alpes, CNRS, TIMC-IMAG Laboratory UMR 5525, Grenoble, France \\ 'INSERM U1062, Nutrition, Obesité et Risque thrombotique, France \\ ${ }^{3}$ Silvermed Institute, Marseille, France
}

*Corresponding author: Stéphane Tanguy, Phd, Université Grenoble Alpes, CNRS, TIMC-IMAG Laboratory UMR 5525, Bâtiment J. Roget, 4ème étage, 38706, La Tronche, France, Tel: (33)-476-637-117, Fax: (33)-476-637-152, E-mail: stephane.tanguy@univ-grenoble-alpes.fr

\begin{abstract}
Introduction: Visceral obesity, a feature of the metabolic syndrome, is associated with metabolic and cardiovascular diseases. Our aim was to study the influence of increased fat intake, a major determinant of central obesity, on Smooth Muscle Cells (SMC) reactivity and its interaction with Perivascular Adipose Tissue (PVAT), which plays an important role in the local regulation of vascular function, with a special emphasis on oxidative stress.
\end{abstract}

Methods: From weaning, rats were fed low-fat or High-Fat Diet (HFD) for 5 months. The implication of oxidative stress mediated by PVAT and/or SMC on vascular function was investigated in isolated endothelium-removed aortic rings.

Results: HFD increased PVAT mass and thiobarbituric acid reactive substances concentrations. Although HFD did not change phenylephrine-induced contraction and PVAT anticontractile activity, the HFD-induced central obesity was associated with increased aortic wall procontractile activity generated by NADPH oxidase. Such phenomenon was counteracted by increased aortic wall dismutation activity and decreased PVAT procontractile activity.

Conclusions: The apparent lack of change of vascular reactivity in HFD-fed rats was subsequent to a new equilibrium state between vascular wall and PVAT oxidative stress-induced procontractile and prorelaxant activities. This observation predicts that any defect in the above-mentioned counterregulatory mechanisms can have deleterious functional consequences.

\section{Keywords}

High-fat diet, Vascular function, Perivascular adipose tissue, Oxidative stress, NADPH oxidase

\begin{abstract}
Abbreviations
AT: Adipose Tissue; BAT: Brown Adipose Tissue; HFD: High-Fat Diet; LFD: Low-Fat Diet; PE: Phenylephrine; PVAT: Perivascular Adipose Tissue; ROS: Reactive Oxygen Species; SMC: Muscle Cells; SOD: Superoxide Dismutase; Tbars: Thiobarbituric Acid Reactive Substances; UCP: Uncoupling Protein; WAT: White Adipose Tissue
\end{abstract}

\section{Introduction}

The metabolic syndrome leads to type 2 diabetes and coronary heart disease, which remains the leading cause of death in developed countries. The metabolic syndrome associates at least three of the following criteria: central obesity, which is characterized by increased fat accumulation at the abdominal visceral level, enhanced circulating triglycerides, decreased HDL-cholesterol, increased arterial blood pressure, and insulin resistance. Central obesity is the major feature of the metabolic syndrome: the Framingham Heart Study, performed in North America between 1988 and 1994, revealed that the metabolic syndrome occurs in $4.6 \%$ of lean subjects, in $22.4 \%$ of patients with overweight, and in $59.6 \%$ of obese patients [1]. As a consequence, obesity, with respect to its continuously growing frequency in developed countries, is becoming a major public health concern. Apart from genetic factors, increased fat intake is known in humans to be, at least in part, responsible for the burden of obesity in developed countries [2]. Therefore, a diet rich in

Citation: Sanchez C, Achard V, Grino M, Tanguy S (2017) Long Term High-Fat Diet-Induced Modification of Vascular Wall and Perivascular Adipose Tissue-Mediated Oxidative Stress: Consequences for Endothelium-Independent Vascular Function in Rats. Int J Clin Cardiol 4:097. doi.org/10.23937/23782951/1410097

Received: April 06, 2017; Accepted: June 22, 2017; Published: June 24, 2017

Copyright: (C) 2017 Sanchez C, et al. This is an open-access article distributed under the terms of the Creative Commons Attribution License, which permits unrestricted use, distribution, and reproduction in any medium, provided the original author and source are credited. 
saturated fatty acids plays an important role in the development and progression of cardiovascular diseases [3]. The mechanisms responsible for diet-associated cardiovascular diseases involve oxidative stress. Indeed, although redox status plays an important role in intracellular signaling pathways under basal conditions, an increased generation of Reactive Oxygen Species (ROS), mainly subsequent to NADPH oxidase and xanthine oxidase activation and antioxidative enzymes reduction, is central in the pathogenesis of cardiovascular disease associated with the metabolic syndrome [4].

Perivascular Adipose Tissue (PVAT) is an ectopic fat depot which plays an important role in regulating vascular function. PVAT exerts a anticontractile effect in both rats and humans [5-7] through an endothelium-dependent mechanism which involves transferable factors such as leptin [8] or angiotensin [7,9] and through an endothelium-independent mechanisms involving $\mathrm{H}_{2} \mathrm{O}_{2}$ [10], $\mathrm{H}_{2} \mathrm{~S}$ [11], NO [12], or palmitic acid methyl ester [13]. The above-mentioned phenomenons can be altered under pathophysiological conditions. Indeed, PVAT from obese subjects with metabolic syndrome is devoid of any anticontractile property [6]. Although diet-induced decrease of endothelium-dependent vasorelaxation is well documented [12,14-16], a direct effect of diet alterations, especially increased fat intake, on vascular smooth muscle cells has received much less attention.

In the present report rats were fed Low-Fat (LFD) or High-Fat Diet (HFD) from weaning and studied at 5 months. Vascular reactivity and the role of PVAT were investigated using Phenylephrine (PE)-stimulated isolated endothelium-removed aortic rings after pharmacological blockade of ROS-synthesizing enzymes or alterations of the dismutation status. The main purpose was to focus on the local interaction between PVAT and SMC in the regulation of ROS production and its consequences on vascular reactivity.

\section{Materials and Methods}

\section{Animals}

All experimental procedures were approved by the Local Animal Care and Use Committee (authorization 13-181). Male Wistar rats, bred in our animal room facility under standard conditions of light (12-h light/dark cycle; lights on at 0600$)$ and temperature $\left(22-24^{\circ} \mathrm{C}\right)$, were fed from weaning with either LFD or HFD (SAFE, Villemoisson-sur-Orge, France) ad libitum with free access to tap water (initial weight 50-60 g) (Supplemental Table 1). As compared to LFD, HFD was characterized by an increase in saturated and monounsaturated fat together with a decrease in polyunsatured fat.

\section{Morphological studies}

Aortic rings including PVAT were fixed in $4 \%$ formaldehyde and embedded in paraffin. Five microns-thick section were opposed onto slides (Superfrost Plus; CML,
Nemours, France) and colored using Masson's trichrome technique. For each slide five crosswise-cut media sections were digitalized using a ProgRes ${ }^{\circledR}$ charge-coupled device camera (Jenoptik GmbH, Jena, Germany) coupled to a DM-RB microscope (Leica, Nanterre, France) at 20X and 63X magnification. For each photomicrograph aortic wall thickness, White (WAT) and Brown Adipose Tissue (BAT) area, WAT and BAT adipocytes surfaces were manually denileated and measured. Averaged values of five images were used for each rat.

\section{Glucose tolerance test}

Fasted five-month-old rats were injected intraperitoneally with $1.5 \mathrm{mg} / \mathrm{g}$ D-glucose (30\% stock solution). Blood samples were drawn under light Aerrane $(2 \%$ in air, Baxter, Maurepas, France) anesthesia by tail venesection before, 30 and $120 \mathrm{~min}$ after the glucose load. Glucose was determined with the Accu-chek performa meter and blood glucose test strips (Roche diagnostics, Roche, Basel, Switzerland). Sampled blood was allowed to clot for $30 \mathrm{~min}$ at room temperature, centrifuged $\left(2600 \times \mathrm{g}\right.$ for $15 \mathrm{~min}$ at $4{ }^{\circ} \mathrm{C}$ ) and the resulting serum was stored at $-80^{\circ} \mathrm{C}$ until assay. Serum insulin was assayed using a commercial radioimmunoassay kit (Millipore, Molsheim, France).

\section{Isolated aortic ring studies}

One week after the glucose tolerance test, rats were anesthetised with sodium pentobarbital (Ceva santé animale, Libourne, France, $60 \mathrm{mg} / \mathrm{kg}$ b.w., intraperitonealIy), the chest was opened, the aorta was rinsed with 10 $\mathrm{ml}$ of heparin $(5 \mathrm{IU} / \mathrm{ml})$ in physiological saline and the descending thoracic aorta was collected in Krebs-Ringer buffer (in mmol/l): $\mathrm{NaCl}: 137.0 ; \mathrm{KCl}: 5.4 ; \mathrm{NaHCO}_{3}: 25.0$; $\mathrm{KH}_{2} \mathrm{PO}_{4}: 1.2 ; \mathrm{MgSO}_{4}: 1.2 ; \mathrm{CaCl}_{2}: 1.2$; glucose: 5.6. Endothelium was removed by gently rubbing the internal surface with fine forceps and successful removal was confirmed by the lack of relaxation in response to carbachol ( $1 \mu \mathrm{mol} / \mathrm{l}$, Sigma, Saint Quentin Fallavier, France) in $1 \mu \mathrm{mol} / \mathrm{I} \mathrm{PE}$ (Sigma)-precontracted rings. Paired aortic rings (4-5 mm long, originating from 6-8 animals/experimental condition) with or without PVAT were incubated at $37^{\circ} \mathrm{C}$ in $5 \mathrm{ml}$ chambers filled with Krebs Ringer bubbled with $95 \% \mathrm{O}_{2}-5 \% \mathrm{CO}_{2}$ gas mixture. A computerized myograph system (EMKA technologies, Paris, France) was used to record the isometric tension of the aortic rings. After equilibration for $90 \mathrm{~min}$ at $2 \mathrm{~g}$ preload, rings were challenged with $56 \mathrm{mmol} / \mathrm{I} \mathrm{KCl}$ twice at an interval of $30 \mathrm{~min}$. Then aortic rings were washed and incubated for $30 \mathrm{~min}$ with the following drugs: tempol $(1 \mathrm{mmol} / \mathrm{l})$, oxypurinol $(100 \mu \mathrm{mol} / \mathrm{l})$, rotenone $(100 \mu \mathrm{mol} / \mathrm{l})$, superoxide dismutase (SOD, $150 \mathrm{IU} / \mathrm{ml}$, Sigma), apocynin (100 $\mu \mathrm{mol} / \mathrm{l})$ or catalase $(1000 \mathrm{IU} / \mathrm{ml}$, Calbiochem, distributed by Merck, Darmstadt, Germany). Then, cumulative doses of $\mathrm{PE}\left(10^{-9}\right.$ to $\left.10^{-5} \mathrm{M}\right)$ were added.

Stock solutions of tempol, oxypurinol, rotenone, and apocynin were diluted in dimethyl sulfoxide. SOD was pre- 
pared in Krebs buffer, whereas catalase was weighed in aliquotes and diluted extemporaneously in Krebs buffer.

\section{RT-PCR analysis}

Aortic rings and their PVAT were carefully dissected immediately after removal, frozen on dry ice and kept at $-80{ }^{\circ} \mathrm{C}$ until analysis. Total RNA was extracted using the guanidine/phenol methodology according to Chomczynski and Sacchi [17] and digested with DNAse (Sigma). RNA concentration was determined spectrophotometrically. RT was performed using $100 \mathrm{ng}$ of RNA and MMLV transcriptase (Promega, Charbonnières, France). 1 pg to $3 \mathrm{ng}$ of RT product (depending upon the cDNA to be measured) were amplified for 40 cycles on a 7300 Real Time PCR system using the SYBR ${ }^{\circ}$ GREEN PCR master mix (Applied Biosystems, Foster City, CA) and specific primers. Relative quantification $(\Delta \mathrm{ct})$ was obtained by normalization against cyclophilin ( $\Delta \mathrm{ct}=\mathrm{ct}$ target gene - ct cyclophilin). The expression level of cyclophylin does not differ between groups. Primers sequences are detailed in Supplemental Table 2.

\section{Thiobarbituric acid reactive substances (Tbars) assay}

Blood samples were obtained by a tail nick from fasted animals lightly anaesthetized with Aerane, centrifuged at $4000 \times \mathrm{rpm}(2000 \mathrm{~g})$ for $15 \mathrm{~min}$ at $4{ }^{\circ} \mathrm{C}$ and the resulting serum was stored at $-80^{\circ} \mathrm{C}$ until assay. The aortic arch and its PVAT were carefully dissected immediately after removal, frozen on dry ice and kept at -80 ${ }^{\circ} \mathrm{C}$ until analysis. Tbars were measured according to Yagi [18]. Tissue protein content was measured using an inhouse bicinchonic acid assay.

\section{Statistical analysis}

Results are expressed as mean \pm SE of tension values above the preload tension. PCR results are expressed as $\%$ of controls. Dose-response data were analyzed and compared (combined effect for EC50 and maximal contraction) by non-linear regression methods using the PRISM software (Graphpad, San Diego, CA). WAT and BAT areas and adipocytes surfaces were compared us ing the Mann-Whitney $U$ test. Body measurements and circulating parameters were compared with the unpaired Student's t test. The Wilcoxon test was used to compare data from the transfer experiments. $\mathrm{P}<0.05$ was considered significant.

\section{Results}

HFD increased PVAT, mesenteric, and epididymal adipose tissue and induced glucose intolerance and insulin resistance

HFD did not change body weight $(540 \pm 12$ vs. $532 \pm$ $12 \mathrm{~g}$ in HFD- or LFD-fed rats, respectively; $\mathrm{P}=0.64$ ), but significantly increased the epididymal and mesenteric fat depots ( $13.43 \pm 0.62$ vs. $10.04 \pm 0.33$ and $11.89 \pm 0.57$ vs. $9.96 \pm 0.44 \mathrm{mg} / \mathrm{g}$ body weight in HFD- or LFD-fed rats, respectively; $\mathrm{P}<0.0001$ and $\mathrm{P}=0.0091$, respectively). Compared with LFD-fed rats, blood glucose and serum insulin levels in fasted HFD-fed animals were increased both under basal conditions and after glucose loading, indicating a state of glucose intolerance and insulin resistance (Table 1). Figure 1 shows counterstained sections of aortic rings obtained from LFD- or HFD-fed rats and the results of the morphological measurements. Aortic wall thickness was not modified by the diet $(137.2 \pm 4.4$ vs. $139.5 \pm 5.4 \mu \mathrm{m}$ in LFD- or HFD-fed rats, respectively; $P=0.64)$. Aortas from LFD-fed animals were surrounded by a thin layer of PVAT, mostly composed of brown adipose tissue (BAT, Figure $1 \mathrm{~A}$ ) in which lipid droplets were small (Figure 1A, upper inset; the lipid droplets were depleted during paraffin embedding and therefore appear as an empty space). Only few areas of White Adipose Tissue (WAT) were observed (Figure 1A, lower inset). Sections obtained from HFD-fed rats showed a thicker PVAT layer (Figure 1B) with enlarged brown (Figure 1B, right inset), and white (Figure 1B, left inset) adipocytes. Microscopic quantification showed that HFD enhanced both BAT and WAT total and adipocytes surfaces. HFD did not induce a significant increase in PVAT leptin or adiponectin mRNA levels $(77.6 \pm 11.8$ and $83.3 \pm 4.1$ vs. $100 \pm 10.9$ and $100 \pm 7.7 \%$ in LFD-fed rats, for leptin and adiponectin $\mathrm{mRNA}$, respectively; $\mathrm{P}>0.05$ ).

\section{HFD has opposite influences on systemic, aortic and PVAT Tbars generation}

Table 2 details the HFD-induced changes in serum and tissue Tbars concentrations. Compared with rats fed LFD, serum, mesenteric and epididymal adipose tissue Tbars levels increased in HFD-fed animals whereas they showed an important decrease in the aortic arch and in the PVAT.

Table 1: Blood glucose and serum insulin during the intraperitoneal glucose tolerance test.

\begin{tabular}{|l|l|l|l|l|}
\hline & Time & LFD (n=18) & HFD (n= 18) & P \\
\hline Blood glucose (mmol/L) & 0 & $6.33 \pm 0.12$ & $7.14 \pm 0.22$ & 0.0024 \\
\hline & 30 & $14.98 \pm 0.63$ & $19.07 \pm 0.54$ & $<0.0001$ \\
\hline AUC (mmol/L/min) & 120 & $8.08 \pm 0.24$ & $9.39 \pm 0.27$ & 0.0009 \\
\hline Serum insulin (pmol/L) & & $11.31 \pm 0.37$ & $13.95 \pm 0.34$ & $<0.0001$ \\
\hline & 0 & $128 \pm 27$ & $317 \pm 39$ & 0.0004 \\
\hline AUC (pmol/L/min) & 30 & $328 \pm 44$ & $896 \pm 46$ & $<0.0001$ \\
\hline
\end{tabular}

Results are expressed as mean \pm SE. 


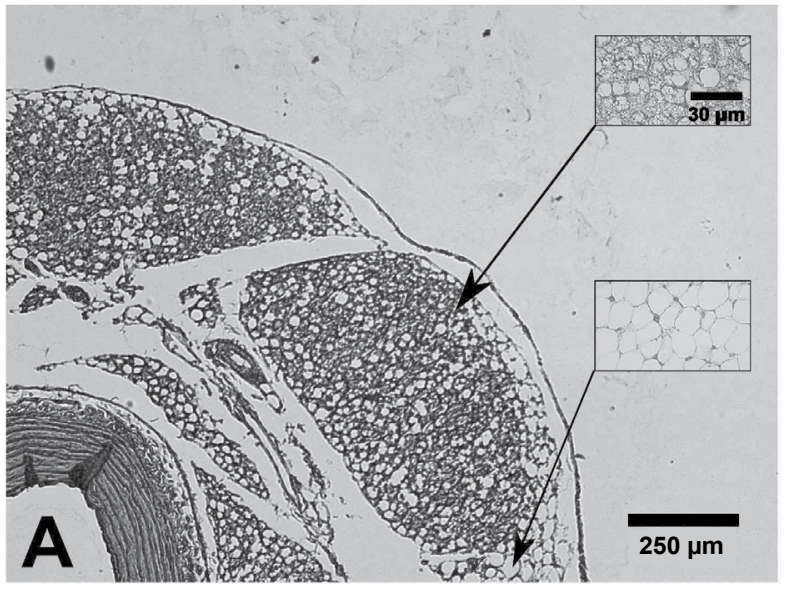

C Total Surface
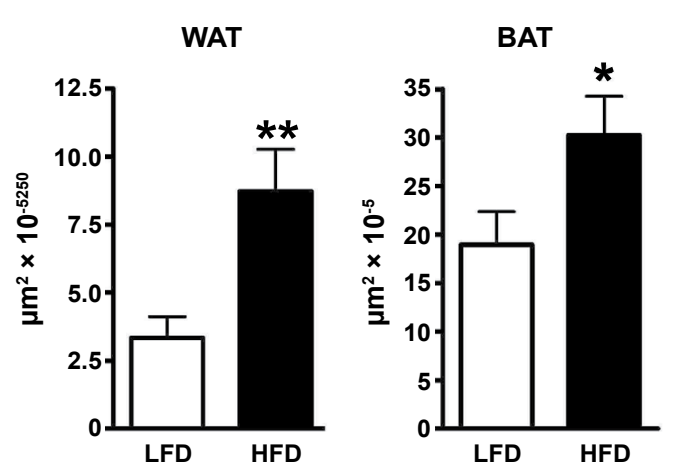

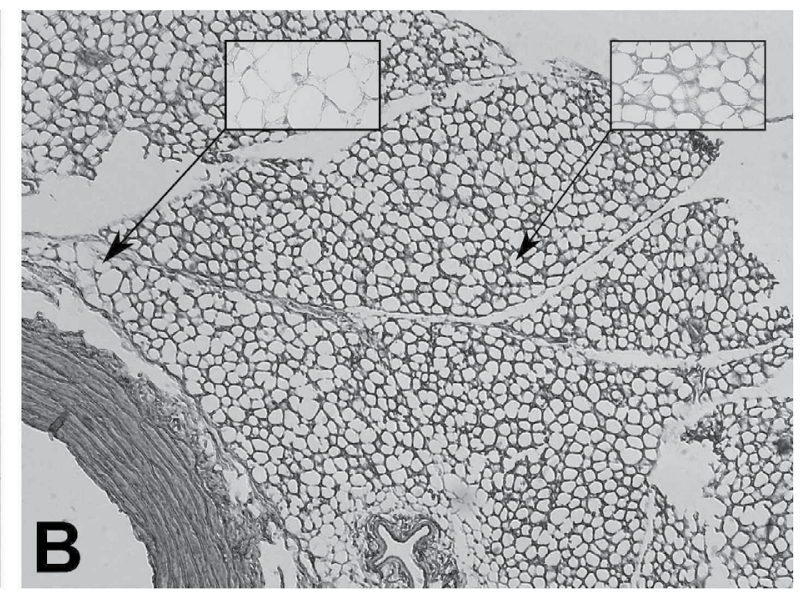

Adipocyte Surface

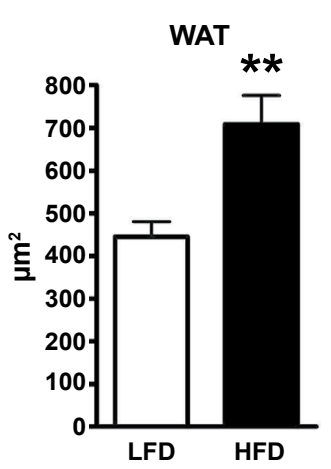

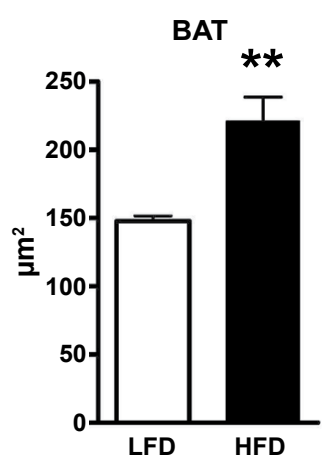

Figure 1: $A, B$ ) Photomicrographs of the aortic wall and the Perivascular Adipose Tissue (PVAT) obtained from Low-Fat (LFD) or High-Fat Diet (HFD) fed rats. Paraffin sections were counterstained with haematoxylin and eosin. Aortas from LFD-fed animals were surrounded by a thin layer of PVAT, mostly composed of BAT (Figure 1A) in which lipid droplets were small (Figure 1A, upper inset; the lipid droplets were depleted during paraffin embedding and therefore appear as an empty space). Only few areas of WAT were observed (Figure 1A, lower inset). Sections obtained from HFD-fed rats showed a thicker PVAT layer (Figure 1B) with enlarged brown (Figure 1B, right inset), and white (Figure 1B, left inset) adipocytes; C: White and brown adipocytes total areas and individual surfaces were measured in counterstained paraffin sections as described in Material and Methods section. Results are expressed as mean \pm SE $(n=10 /$ group for aortic wall thickness, overall WAT and BAT total areas and WAT adipocytes area, $n=6 /$ group for BAT adipocytes surfaces WAT: White Adipose Tissue; BAT: Brown Adipose Tissue).

Table 2: Thiobarbituric acid reactive substances in serum and in various tissues obtained from rats fed from weaning with LowFat (LFD) or High-Fat (HFD) Diet.

\begin{tabular}{|c|c|c|c|}
\hline & \multicolumn{2}{|l|}{ Diet } & \multirow[t]{2}{*}{$\mathbf{P}$} \\
\hline & LFD $(n=20)$ & $\operatorname{HFD}(n=16)$ & \\
\hline Serum $(\mu \mathrm{mol} / \mathrm{l})$ & $1.06 \pm 0.04$ & $1.24 \pm 0.06$ & 0.036 \\
\hline \multicolumn{4}{|c|}{ Tissues (nmol/mg protein) } \\
\hline Aortic arch & $11.20 \pm 0.75$ & $3.69 \pm 0.46$ & $<0.0001$ \\
\hline Perivascular AT & $0.44 \pm 0.05$ & $0.21 \pm 0.04$ & 0.0012 \\
\hline Mesenteric AT & $1.13 \pm 0.19$ & $1.81 \pm 0.24$ & 0.017 \\
\hline Epididymal AT & $1.96 \pm 0.30$ & $3.22 \pm 0.36$ & 0.0027 \\
\hline
\end{tabular}

Results are expressed as mean \pm SE. AT: adipose tissue.

\section{PVAT plays an anticontractile role, independently of PE}

When aortic rings devoid of PVAT were incubated with a depolarizing dose of $\mathrm{KCl}$, maximal contraction was not statistically different between LFD- or HFD-fed rats $(4.0 \pm 0.25$ vs. $4.01 \pm 0.23 \mathrm{~g}, \mathrm{P}=0.93)$. The presence of PVAT decreased the $\mathrm{KCl}$-induced maximal contraction (3.14 \pm 0.23 and $3.22 \pm 0.27 \mathrm{~g}$, in LFD- or HFD-fed rats, respectively; $P=0.0172$ and $P=0.033$ ) to the same extent in rings obtained from both groups $(P=0.82)$.

\section{HFD induced NADPH-dependent generation of procontractile $\mathrm{O2}^{\circ}$ - in the aortic wall (Figure 2)}

In rings without PVAT, preincubation with tempol, a ROS scavenging reagent, decreased the response to $P E$, this effect being more important in HFD-fed rats compared with LFD-fed animals $(-59 \%$ and $-24 \%$, respectively). Preincubation with apocynin, a NADPH oxidase inhibitor, was without effect in rings obtained from LFD-fed rats and reduced the contraction to PE in rings obtained from HFD-fed animals. Preincubation 
LFD
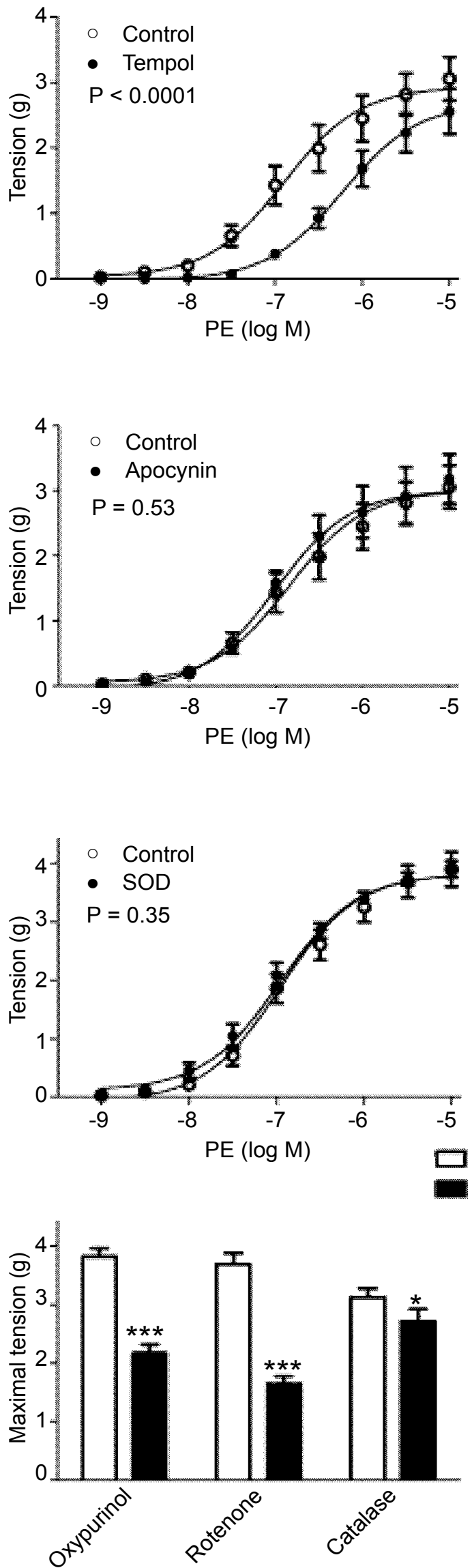

HFD
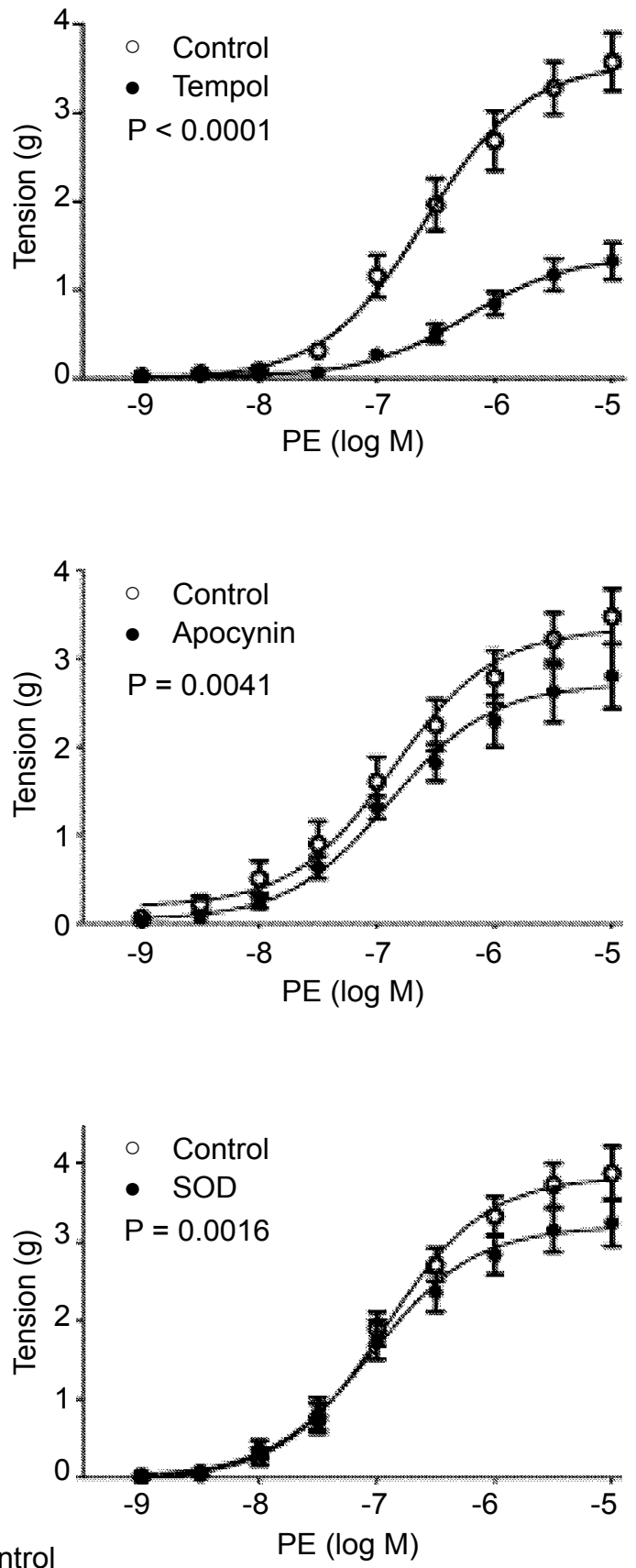

Treatment

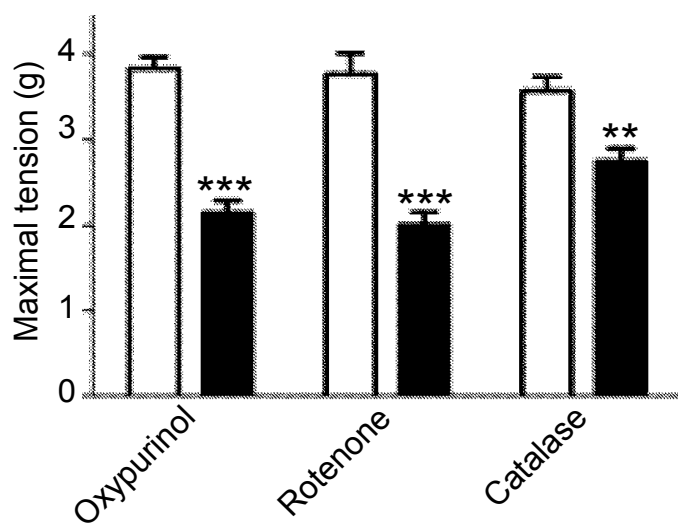

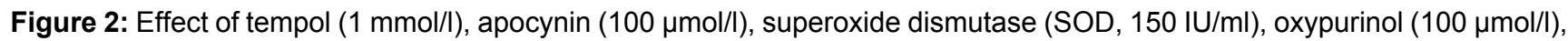
rotenone $(100 \mu \mathrm{mol} / \mathrm{l})$, catalase $(1000 \mathrm{IU} / \mathrm{ml})$, on cumulative Phenylephrine $(\mathrm{PE})$-induced contraction of endothelium-denuded aortic rings without PVAT obtained from rats fed Low-Fat (LFD) or High-Fat Diet (HFD). $n=6 / 8$ per group. Results are expressed as mean \pm SE. Statistical analysis was performed using non-linear regression methods. For a matter of clarity only the maximal effect is shown in the lowest panel ${ }^{*}: P<0.05 ;{ }^{*}$ : $P<0.01$; ${ }^{* * *}: P<0.001$. 
with SOD was devoid of effect in LFD rings rats, whereas it decreased the contraction to PE in HFD rings. Taken together, the above-mentioned observations demonstrate that the increased procontractile activity found in HFD rings was, at least in part, generated by NADPH oxidase through $02^{*}$. The contractile response to $\mathrm{PE}$ was attenuated by oxypurinol, a xanthine oxidase inhibitor, or rotenone, a cytochrome c oxidase inhibitor, in both groups ( -40 and $-54 \%$ in LFD, and -40 and $-44 \%$ in HFD). Catalase markedly attenuated the contractile response to $P E$, to the same extent in rings obtained from LFDor HFD-fed rats $(-25 \%$ in LFD and $-17 \%$ in HFD). These findings indicate that aortic wall xanthine oxidase and cytochrome c oxidase have procontractile properties, mediated by $\mathrm{H}_{2} \mathrm{O}_{2}$.

\section{PVAT-synthesized $\mathrm{H}_{2} \mathrm{O}_{2}$ is involved in the anticon- tractile properties of PVAT}

When aortic rings were incubated with increasing doses of PE, maximal contraction and EC50 were not different between LFD- or HFD-fed rats (3.50 \pm 0.11 vs. $3.60 \pm 0.11 \mathrm{~g}$ and $0.061 \pm 0.012$ vs. $0.058 \pm 0.010 \mu \mathrm{mol} / \mathrm{I}$ in rings obtained from LFD- or HFD-fed rats, $\mathrm{P}=0.96$ and $P=0.86$, respectively). The presence of PVAT decreased $P E$-induced maximal contraction $(P<0.0001)$ to the same extent in rings obtained from LFD- or HFD-fed rats $(2.97 \pm 0.11$ and $3.06 \pm 0.11 \mathrm{~g}$ in LFD- or HFD-fed rats, respectively; $P=0.77)$, and the EC50 for $P E$ was not changed $(0.091 \pm 0.017$ and $0.082 \pm 0.015 \mu \mathrm{mol} / \mathrm{l}$ in LFDor HFD-fed rats, respectively; $\mathrm{P}=0.73$ ). Preincubation with catalase suppressed the anticontractile properties of PVAT, demonstrating that this effect was mediated through $\mathrm{H}_{2} \mathrm{O}_{2}$ (Figure 3).

\section{HFD modulates procontractile and prorelaxant ROS generation in PVAT (Figure 3)}

The presence of PVAT and the diet modified the effect of the various drugs tested. We found that, in LFD rats, the prorelaxant properties of PVAT were increased after preincubation with tempol, demonstrating that, under our experimental conditions, PVAT has a ROS-mediated procontractile activity. The response to PE after preincubation with tempol in rings with PVAT compared to rings without PVAT was reduced in HFD-fed rats compared with LFD-fed animals (-10\% and $-26 \%$, respectively). Preincubation with apocynin supressed the anticontractile properties of PVAT in rings obtained from LFD-fed rats whereas it was devoid of effect in rings obtained from HFD-fed animals. Incubation with oxypurinol amplified the anticontractile effect of PVAT in LFD animals while it reversed this effect in HFD rats. This suggests that $\mathrm{O}^{\circ}$ - generated by PVAT xanthine oxidase acts as procontractile ROS in LFD animals while, after dismutation to $\mathrm{H}_{2} \mathrm{O}_{2}$, it shifts to anticontractile in HFD animals. In rings obtained from LFD animals, incubation with rotenone reversed the anticontractile effect of PVAT whereas it was devoid of effect in HFD rats. Such data indicates that PVAT cytochrome c oxidase is anticontractile in LFD rats whereas it is no longer active in HFD animals. Incubation with SOD increased the anticontractile effect of PVAT in LFD rats whereas it was devoid of effect in HFD animals. Catalase-induced $\mathrm{H}_{2} \mathrm{O}_{2}$ removal suppressed the effect of PVAT in both groups. The above-mentioned observations are consistent with a $\mathrm{H}_{2} \mathrm{O}_{2}$-mediated anticontractile mechanism.

\section{HFD increases the mRNAs coding for NADPH and xanthine oxidase in the aortic wall}

The results of the various RT-PCR analyses are shown in Table 3. In the aortic wall HFD increased the mRNAs coding for three NADPH oxidase subunits (p47phox, p67phox, and NOX4), xanthine oxidase and the dismutation enzymes (SOD and catalase). In PVAT, HFD increased the mRNAs coding for cytochrome c oxidase, glutathione peroxidase and UCP and it decreased those coding for p22phox, without any significant change in the other NADPH subunits.

\section{Discussion}

Our data show that HFD increased mesenteric and epididymal fat depots without any change in body weight. This observation indicates that HFD-fed rats had decreased lean mass, most probably subsequent to the early onset of the diet. In addition HFD modulated PVAT phenotype with increased BAT and WAT depots. We show that feeding rats with HFD was followed by increased systemic oxidative stress, as evidenced by enhanced circulating and mesenteric and epididymal Tbars levels. Such changes in oxidative stress could be subsequent to changes in glucose metabolic status, with glucose intolerance and insulin resistance [19]. However, the main result of the present study is that HFD has more complex influences on both the aortic wall and PVAT oxidative stress levels leading to modifications of endothelium-independent vascular wall reactivity.

\section{HFD increases vascular wall procontractile potency}

In the aortic wall of rings obtained from LFD-fed rats, ROS generated by xanthine oxidase and cytochrome c oxidase had vasocontractile effects. Our results suggest that this vasomotor effect was mediated by $\mathrm{H}_{2} \mathrm{O}_{2}$. Such ROS generating mechanisms were also effective in HFD-fed animals. However, the larger negative effect of tempol in HFD rings compared with LFD suggests that, in HFD animals, the above-mentioned ROS-producing enzymes are more active or that ROS emanate from another source. This hypothesis can be partly explained by our observation showing that xanthine oxidase mRNA levels were increased in the aortic wall of HFD-fed rats. This observation is consistent with the report of Erdei, et al. [15] who showed that HFD induced increased xanthine oxidase activity in the carotid arteries. HFD-induced increased aortic wall NADPH oxidase-dependent ROS generation could also participate in the abovementioned phenomenon. Indeed, we demonstrate that apocynin, which was devoid of effects in rings obtained 


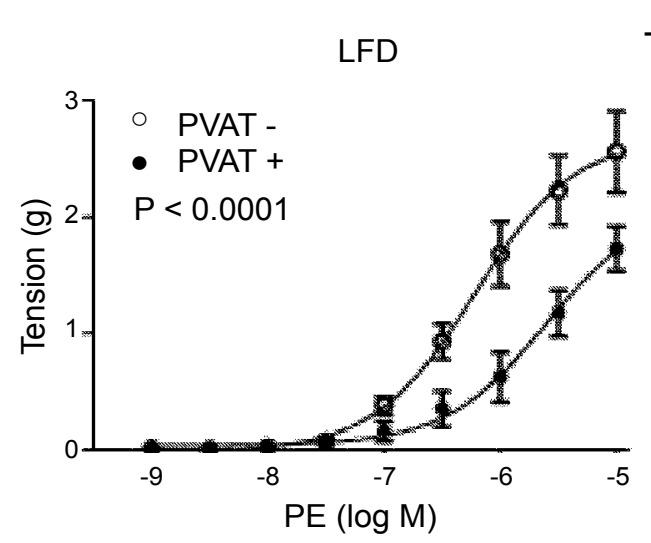

Tempol

HFD

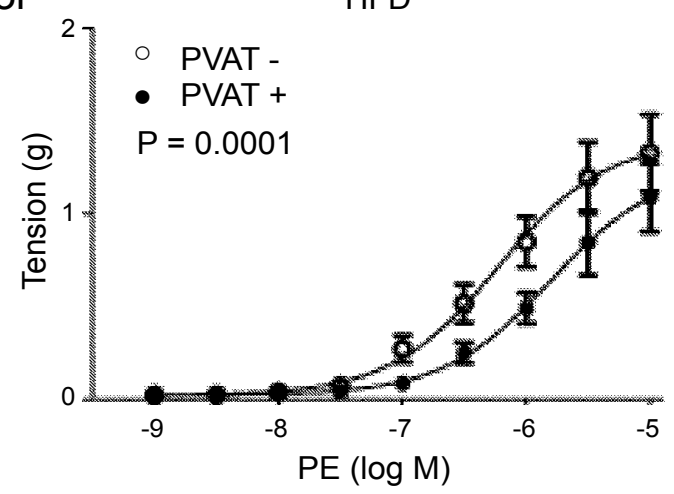

Oxypurinol
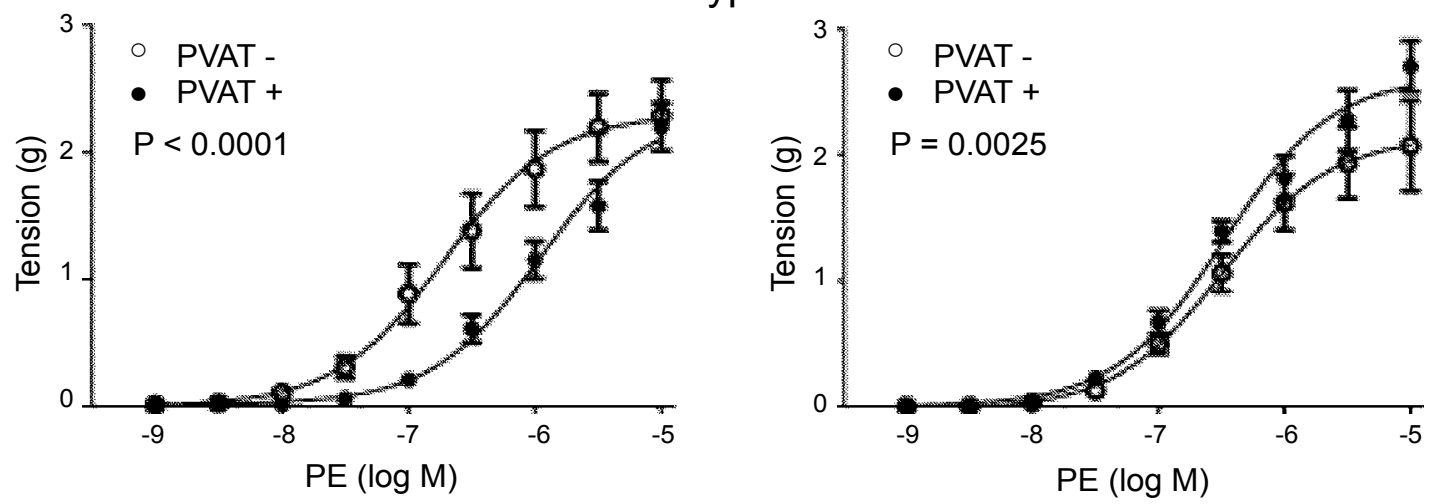

Rotenone
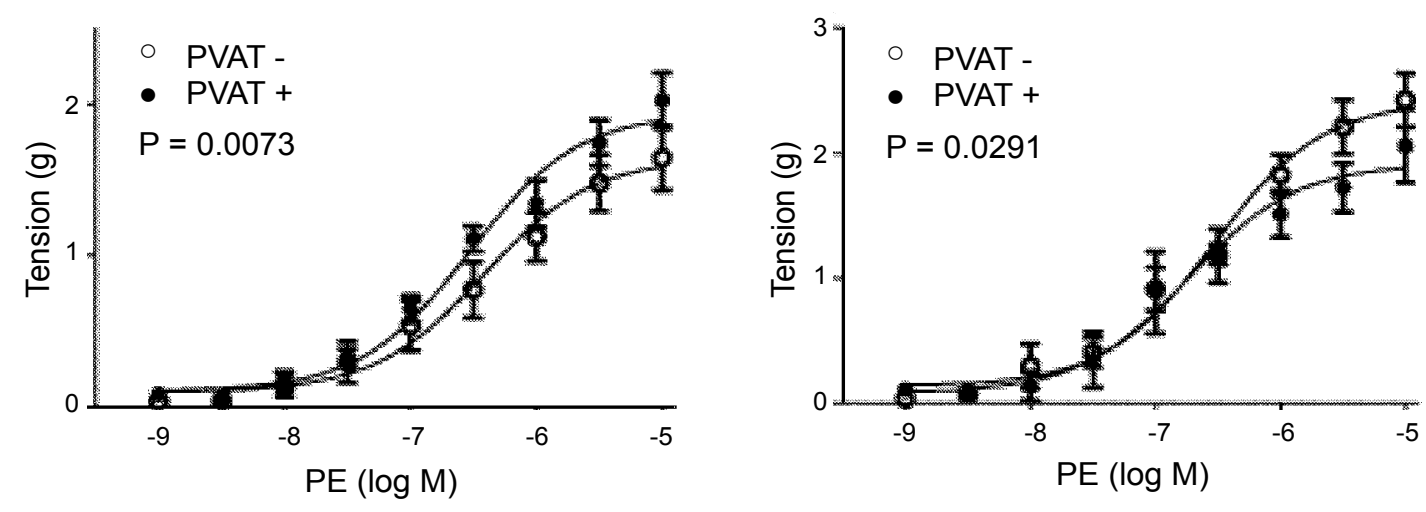

PVAT -

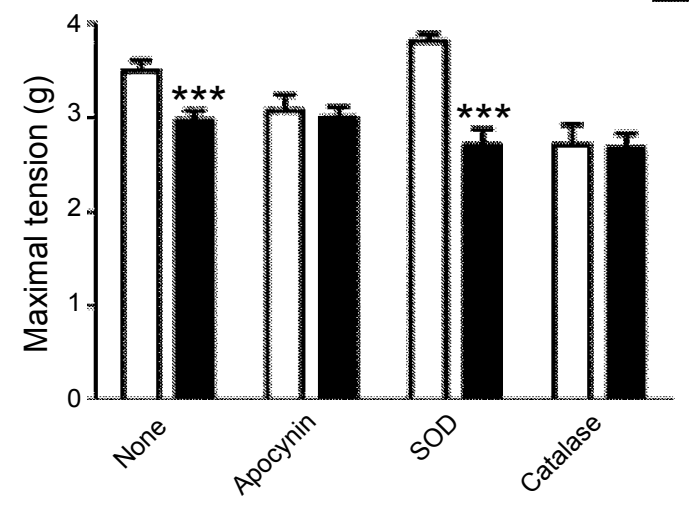

PVAT +

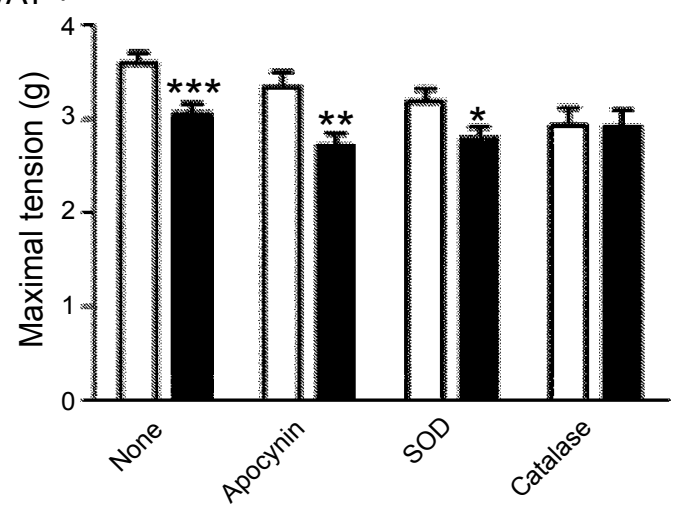

Treatment

Figure 3: Effect of tempol ( $1 \mathrm{mmol} / \mathrm{l})$, oxypurinol $(100 \mu \mathrm{mol} / \mathrm{l})$, rotenone $(100 \mu \mathrm{mol} / \mathrm{l})$, apocynin $(100 \mu \mathrm{mol} / \mathrm{l})$, Superoxide Dismutase (SOD, $150 \mathrm{IU} / \mathrm{ml})$, and catalase $(1000 \mathrm{IU} / \mathrm{ml})$, on cumulative Phenylephrine (PE)-induced contraction of endothelium-denuded aortic rings without or with PVAT (PVAT- and PVAT+, respectively) obtained from rats fed Low-Fat (LFD) or High-Fat Diet (HFD). $n=6 / 8$ per group. Results are expressed as mean \pm SE. Statistical analysis was performed using non-linear regression methods. For a matter of clarity only the maximal effect is shown in the lowest panel ${ }^{*}$ : $\mathrm{P}<0.05$; ${ }^{* *}$ : $\mathrm{P}<0.01$; ${ }^{* *}$ : $\mathrm{P}<0.001$. 
Table 3: Expression of the various mRNAs involved in reactive oxygen species and $\mathrm{H}_{2} \mathrm{O}_{2}$ generation and dismutation in aortic wall and Perivascular Adipose Tissue (PVAT) obtained from rats fed from weaning with Low-Fat (LFD, $n=20)$ or High-Fat Diet (HFD, $n=16)$.

\begin{tabular}{|c|c|c|c|c|c|c|}
\hline Aortic wall & & & & PVAT & & \\
\hline mRNA & Diet & & $\mathrm{P}$ & Diet & & $\mathrm{P}$ \\
\hline & LFD & HFD & & LFD & HFD & \\
\hline p22phox & $100.0 \pm 7.1$ & $83.1 \pm 6.7$ & 0.1 & $100.0 \pm 8.6$ & $70.8 \pm 5.7$ & 0.0096 \\
\hline p40phox & $100.0 \pm 15.9$ & $108.6 \pm 16.4$ & 0.71 & $100.0 \pm 9.7$ & $81.3 \pm 7.5$ & 0.14 \\
\hline p47phox & $100.0 \pm 11.1$ & $159.0 \pm 21.4$ & 0.014 & $100.0 \pm 8.8$ & $91.5 \pm 6.9$ & 0.46 \\
\hline p67phox & $100.0 \pm 9.7$ & $131.9 \pm 11.6$ & 0.041 & $100.0 \pm 10.2$ & $73.6 \pm 9.5$ & 0.068 \\
\hline NOX2 & $100.0 \pm 9.9$ & $92.3 \pm 9.7$ & 0.59 & $100.0 \pm 10.6$ & $74.2 \pm 7.2$ & 0.06 \\
\hline NOX4 & $100.0 \pm 7.2$ & $129.9 \pm 11.0$ & 0.025 & $100.0 \pm 15.2$ & $80.1 \pm 8.4$ & 0.29 \\
\hline Xanthine oxidase & $100.0 \pm 7.0$ & $143.2 \pm 11.9$ & 0.0027 & $100.0 \pm 12.2$ & $92.1 \pm 10.4$ & 0.63 \\
\hline Cytochrome c oxidase subunit I & $100.0 \pm 7.4$ & $98.8 \pm 9.2$ & 0.92 & $100.0 \pm 7.6$ & $171.9 \pm 13.4$ & $<0.0001$ \\
\hline Cytochrome c oxidase subunit II & $100.0 \pm 16.6$ & $80.2 \pm 11.8$ & 0.34 & $100.0 \pm 7.7$ & $179.7 \pm 20.4$ & 0.0004 \\
\hline Cytochrome c oxidase subunit III & $100.0 \pm 7.6$ & $98.7 \pm 11.6$ & 0.92 & $100.0 \pm 7.1$ & $171.5 \pm 15.1$ & $<0.0001$ \\
\hline SOD-1 & $100.0 \pm 5.3$ & $163.9 \pm 12.3$ & $<0.0001$ & $100.0 \pm 7.5$ & $84.6 \pm 3.7$ & 0.086 \\
\hline Catalase & $100.0 \pm 9.6$ & $146.2 \pm 10.2$ & 0.0023 & $100.0 \pm 8.2$ & $96.8 \pm 5.1$ & 0.75 \\
\hline UCP-1 & & & & $100.0 \pm 18.1$ & $208.2 \pm 12.8$ & $<0.0001$ \\
\hline UCP-2 & & & & $100.0 \pm 7.8$ & $100.3 \pm 7.4$ & 0.98 \\
\hline UCP-3 & & & & $100.0 \pm 13.1$ & $157.8 \pm 11.8$ & 0.0026 \\
\hline
\end{tabular}

Results (mean $\pm \mathrm{SE}$ ) are expressed as the percentage of LFD. SOD: Superoxide Dismutase; UCP: Uncoupling Protein.

from LFD rats, decreased the response to $P E$ in rings obtained from HFD-fed animals. Increased NADPH oxidase-dependent ROS generation can be explained by the upregulation of p67phox mRNA, which is a cytosolic activator for NOX1 and NOX2 [20]. The negative effect of SOD on PE-induced contraction found in HFD rings confirms that $\mathrm{O} 2 \cdot-$ mediated this procontractile potency. These results give further information concerning the triggers of HFD effect, when compared with previous data from Bourgoin, et al. [14] and Ketonen, et al. [16] who have reported that HFD induced an increase in aortic wall gp91phox (NOX2) protein and mRNA levels. In addition, the enhanced procontractile potency of the aortic wall of HFD rats could be subsequent to increased $\mathrm{H}_{2} \mathrm{O}_{2}$ generation. Indeed, NOX4, whose mRNA was increased in the aortic wall of HFD-fed rats, generates $\mathrm{H}_{2} \mathrm{O}_{2}$ rather than $\mathrm{O}^{\circ}-$ as its primary ROS $[20,21]$. However, we found that PE-induced contraction in rings without PVAT was comparable between LFD and HFD rats. This apparent discrepancy can be explained by an elevated dismutation activity, as evidenced by the increase in SOD and catalase mRNA concentrations. This hypothesis is supported by our findings showing that aortic arch Tbars levels were strongly decreased in HFDfed rats. Thus, the above-mentioned changes could be related to a counterregulatory phenomenon aimed at protecting the vessel from an increased ROS generation.

\section{The anticontractile properties of PVAT are mediated through $\mathrm{H}_{2} \mathrm{O}_{2}$ and altered by HFD}

We found, in agreement with previously published studies $[5,10]$, that PVAT has anticontractile properties mediated by $\mathrm{H}_{2} \mathrm{O}_{2}$, this effect being comparable between LFD and HFD animals. In addition, our study provides new, original data regarding the characterization of the ROS sources involved in the anticontractile properties of PVAT and their regulation after HFD. In- deed, we demonstrate that this anticontractile activity was, at least in part, mediated by NADPH oxidase and cytochrome c oxidase. In rings obtained from LFD-fed rats, the prorelaxant activity of PVAT disappeared after incubation with apocynin. This phenomenon was not found in rings obtained from HFD-fed animals. Our RTPCR measurements suggest that the above-mentioned phenomenon could be subsequent to a down-regulation of PVAT p22phox. The integral membrane protein p22phox forms a heterodimeric enzyme complex with NADPH oxidases and is required for their catalytic activity. In particular p22phox is an important regulator of NOX4 activity [22].

\section{HFD reduces PVAT procontractile activity}

In addition, PVAT from LFD rats had also a procontractile activity, as evidenced by the reduced PE-induced contraction found after preincubation with tempol. Under our experimental conditions, this procontractile potency implicated $02^{\circ}$ - generated by xanthine oxidase. This observation confirms and extends the findings by Gao, et al. [23] who showed that, in response to peripheral nerve activation by electrical field stimulation, PVAT enhances arterial contractility through the production of $2^{-}$- mediated by NADPH oxidase. In this context our findings clearly demonstrate that in HFD rats the procontractile activity of PVAT decreased. The anticontractile potency of ROS generated by cytochrome c oxidase disappeared and was replaced by a procontractile activity mediated through $02^{-}$-. This was accompanied by an upregulation of the mRNAs coding for the cytochrome c oxidase subunits. However, the large increase (about $70 \%$ ) in the various subunits of cytochrome c oxidase mRNAs did not parallel the reduction of PE-induced contraction after incubation with rotenone (-17\%). Such discrepancy can be explained by the concomitant in- 
crease in UCP-3 mRNA since it is established that activation of UCP-3 induces proton leak, thus providing a negative feedback loop for mitochondrial ROS production [24]. Conversely, the procontractile role of ROS generated by xanthine oxidase was replaced by an anticontractile action mediated by $\mathrm{H}_{2} \mathrm{O}_{2}$. As a consequence, the net effect of the above-mentioned changes in ROS production resulted in a decrease of $\mathrm{PE}$-induced procontractile potency of PVAT compared with LFD, as attested by the reduced negative effect of tempol on the PE-induced contraction. Finally, this hypothesis is consistent with the reduction of Tbars levels in PVAT in HFD rats compared with LFD animals. Taken together, all the above mentioned alterations of ROS production and oxidative stress generation can represent additional counterregulatory mechanisms in PVAT in order to compensate for an increased procontractile ROS generation in the aortic wall.

In conclusion our data show that this rodent model of diet-induced central obesity and insulin resistance was associated with increased procontractile activity in the aortic wall. Such phenomenon was counteracted by enhanced dismutation activity in the aortic wall and decreased procontractile activity provided by the PVAT. As a consequence, these observations predict that any defect in the above-mentioned counterregulatory mechanisms can have deleterious functional consequences.

\section{Funding}

This work was supported by grants from the Fondation "Santé, Sport et Développement Durable" Aix-Marseille University.

\section{Duality of Interest}

No competing financial interests exist.

\section{Conflict of Interest}

No conflict of interest.

\section{Contribution Statement}

All authors substantially contributed to conception and design of the study, acquisition of data or analysis and interpretation of data, drafted the article or revised it critically for important intellectual content and approved of the version to be published.

\section{References}

1. Park YW, Zhu S, Palaniappan L, Heshka S, Carnethon MR, et al. (2003) The metabolic syndrome: prevalence and associated risk factors findings in the US population from the third National Health and Nutrition examination survey, 1988-1994. Arch Intern Med 163: 427-436.

2. Hill JO, Melanson EL, Wyatt HT (2000) Dietary fat intake and regulation of energy balance: implications for obesity. $J$ Nutr 130: 284S-288S.

3. Vafeiadou K, Weech M, Sharma V, Yaqoob P, Todd S, et al. (2012) A review of the evidence for the effect of total dietary fat, saturated, monounsaturated and $n-6$ polyunsaturated fatty acids on vascular function, endothelial progenitor cells and microparticles. Br J Nutr 107: 303-324.
4. Otani H (2011) Oxidative stress as pathogenesis of cardiovascular risk associated with metabolic syndrome. Antioxid Redox Signal 15: 1911-1926.

5. Gao YJ, Zeng ZH, Teoh K, Sharma AM, Abouzahr L, et al. (2005) Perivascular adipose tissue modulates vascular function in the human internal thoracic artery. J Thorac Cardiovasc Surg 130: 1130-1136.

6. Greenstein AS, Khavandi K, Withers SB, Sonoyama K, Clancy O, et al. (2009) Local inflammation and hypoxia abolish the protective anticontractile properties of perivascular fat in obese patients. Circulation 119: 1661-1670.

7. Soltis EE, Cassis LA (1991) Influence of perivascular adipose tissue on rat aortic smooth muscle responsiveness. Clin Exp Hypertens A 13: 277-296.

8. Lembo G, Vecchione C, Fratta L, Marino G, Trimarco V, et al. (2000) Leptin induces direct vasodilation through distinct endothelial mechanisms. Diabetes 49: 293-297.

9. Lee RM, Lu C, Su LY, Gao YJ (2009) Endothelium-dependent relaxation factor released by perivascular adipose tissue. J Hypertens 27: 782-790.

10. Gao YJ, Lu C, Su LY, Sharma AM, Lee RM (2007) Modulation of vascular function by perivascular adipose tissue: the role of endothelium and hydrogen peroxide. $\mathrm{Br} \mathrm{J}$ Pharmacol 151: 323-331.

11. Schleifenbaum J, Köhn C, Voblova N, Dubrovska G, Zavarirskaya O, et al. (2010) Systemic peripheral artery relaxation by KCNQ channel openers and hydrogen sulfide. J Hypertens 28: 1875-1882.

12. Gil-Ortega M, Stucchi P, Guzmán-Ruiz R, Cano V, Arribas $S$, et al. (2010) Adaptative nitric oxide overproduction in perivascular adipose tissue during early diet-induced obesity. Endocrinology 151: 3299-3306.

13. Lee YC, Chang $\mathrm{HH}$, Chiang $\mathrm{CL}$, Liu $\mathrm{CH}$, Yeh JI, et al. (2011) Role of perivascular adipose tissue-derived methyl palmitate in vascular tone regulation and pathogenesis of hypertension. Circulation 124: 1160-1171.

14. Bourgoin F, Bachelard H, Badeau M, Mélançon S, Pitre M, et al. (2008) Endothelial and vascular dysfunctions and insulin resistance in rats fed a high-fat, high-sucrose diet. Am J Physiol Heart Circ Physiol 295: 1044-1055.

15. Erdei N, Tóth A, Pásztor ET, Papp Z, Edes I, et al. (2006) High-fat diet-induced reduction in nitric oxide-dependent arteriolar dilation in rats: role of xanthine oxidase-derived superoxide anion. Am J Physiol Heart Circ Physiol 291: 2107-2115.

16. Ketonen J, Shi J, Martonen E, Mervaala E (2010) Periadventitial adipose tissue promotes endothelial dysfunction via oxidative stress in diet-induced obese $\mathrm{C} 57 \mathrm{BI} / 6$ mice. Circ J 74: 1479-1487.

17. Chomczynski P, Sacchi N (1987) Single-step method of RNA isolation by acid guanidinium thiocyanate-phenol-chloroform extraction. Anal Biochem 162: 156-159.

18. Yagi K (1998) Simple assay for the level of total lipid peroxides in serum or plasma. Methods Mol Biol 108: 101-106.

19. Evans JL, Goldfine ID, Maddux BA, Grodsky GM (2002) Oxidative stress and stress-activated signaling pathways: a unifying hypothesis of type 2 diabetes. Endocr Rev 23: 599-622.

20. Dikalov SI, Dikalova AE, Bikineyeva AT, Schmidt HH, Harrison DG, et al. (2008) Distinct roles of Nox1 and Nox4 in basal and angiotensin II-stimulated superoxide and hydrogen peroxide production. Free Radic Biol Med 45: 13401351. 
21. Drummond GR, Selemidis S, Griendling KK, Sobey CG (2011) Combating oxidative stress in vascular disease: NADPH oxidases as therapeutic targets. Nat Rev Drug Discov 10: $453-471$.

22. von Löhneysen $K$, Noack $D$, Jesaitis AJ, Dinauer MC, Knaus UG (2008) Mutational analysis reveals distinct features of the Nox4-P22 phox complex. J Biol Chem 283: 35273-35282.

23. Gao YJ, Takemori K, Su LY, An WS, Lu C, et al. (2006) Perivascular adipose tissue promotes vasoconstriction: the role of superoxide anion. Cardiovasc Res 71: 363-373.

24. Mailloux RJ, Harper ME (2011) Uncoupling proteins and the control of mitochondrial reactive oxygen species production. Free Radic Biol Med 51: 1106-1115.
Supplemental Table 1: Nutriment and fatty acid composition of Low Fat (LFD) and High Fat (HFD) Diet (fatty acids are expressed in $\%$ relative to total fatty acids).

\begin{tabular}{|l|l|l|}
\hline & LFD & HFD \\
\hline Energy (kcal/g) & 3.2 & 5.0 \\
\hline Lipids (\%) & 3.1 & 0 \\
\hline Lipids as lard (\%) & 0 & 30.0 \\
\hline Proteins (\%) & 16.1 & 16.1 \\
\hline Carbohydrates as corn starch (\%) & 23.0 & 0 \\
\hline Carbohydrates as saccharose (\%) & 0 & 37.0 \\
\hline Vitamins and minerals (\%) & 5.0 & 5.0 \\
\hline Moisture (\%) & 15.8 & 11.9 \\
\hline Fatty acids (\% relative) & & \\
\hline C14:0 & 0.6 & 1.4 \\
\hline C15:0 & 0.1 & 0.1 \\
\hline C16:0 & 19.3 & 24.4 \\
\hline C16:1 & 0.7 & 2.5 \\
\hline C17:0 & 0.1 & 0.3 \\
\hline C17:1 & 0.1 & 0.3 \\
\hline C18:0 & 2.1 & 12.8 \\
\hline C18:1 & 18.5 & 40.1 \\
\hline C18:2n-6 & 49.6 & 10.5 \\
\hline C18:3n-3 & 4.1 & 0.9 \\
\hline C20:1 & 1.0 & 0.8 \\
\hline C20:4n-6 & 0.2 & 0.2 \\
\hline C20:5n-3 & 0.5 & nd \\
\hline C22:1 & 0.7 & nd \\
\hline Saturated fat & 23.0 & 39.6 \\
\hline Monounsaturated fat & 21.4 & 47.9 \\
\hline Polyunsaturated fat & 55.7 & 12.8 \\
\hline Omega 3 Polyunsaturated fat & 5.7 & 1.1 \\
\hline Omega 6 Polyunsaturated fat & 49.8 & 11.4 \\
\hline & & \\
\hline
\end{tabular}

nd: Not Detectable.

Supplemental Table 2: Nucleotide sequences of primers used for the qRT-PCR.

\begin{tabular}{|c|c|c|}
\hline mRNA & Forward primer & Reverse primer \\
\hline p22phox & 5'-ATGTGGGCCAACGAACAG-3' & 5'-CCAAAGTACCACTGCGTGAA-3' \\
\hline p40phox & 5'-GAGGAGAAGAGGGGCTTCAC-3' & 5'-CGATATCGGCGGTAGATGAG-3' \\
\hline p47phox & 5'-CACCTTCATTCGCCACATC-3' & 5'-GTCCTGCCACTTAACCAGGA-3' \\
\hline p67phox & 5'-GCTGCATGTACACCATCCTG-3' & 5'-GGAAGTAAGCCACTGCCAAG-3' \\
\hline NOX2 & 5'-TGATCTTGCTGCCAGTGTGT-3' & 5'-GTTCCTGTCCAGTTGTCTTCG-3' \\
\hline NOX4 & 5'-TCCCTCAGATGTCATGGAACT-3' & 5'-TGCTGATACACTGGGACAATG-3' \\
\hline Cyt c ox-I & 5'-CCTGATATAGCATTCCCACGA-3' & 5'-CTGTTCCAGCTCCAGCTTCT-3' \\
\hline Cyt c ox-II & 5'-GCTTACAAGACGCCACATCA-3' & 5'-CTAGGGAGCTGATGAGGAATACA-3' \\
\hline Cyt c ox-III & 5'-GGCCTCCGATACGGAATAAT-3' & 5'-TGGGTAGGAACTAGGCTGGA-3' \\
\hline SOD-1 & 5'-CCACTGCAGGACCTCATTTT-3' & 5'-CTTTCCAGCAGCCACATTG-3' \\
\hline Catalase & 5'-AGGCAAAGGTGTTTGAGCAT-3' & 5'-GAGGGTCACGAACTGTGTCA-3' \\
\hline UCP-1 & 5'-AATCAGCTTTGCTTCCСTCA-3' & 5'-CGAGATCTTGCTTCCCAAAG-3' \\
\hline UCP-2 & 5'-CAGTTCTACACCAAGGGCTCA-3' & 5'-ATCTGTAGGTTGGGCCACAG-3' \\
\hline UCP-3 & 5'-TTGTGCTGAGATGGTGACCT-3' & 5'-AGCTCCAAAGGCAGAGACAA-3' \\
\hline Adiponectin & 5'-TGGTCACAATGGGATACCG-3' & 5'-CATCTCCTGGGTCACCCTTA-3' \\
\hline Leptin & 5'-CAAGACCATTGTCACCAGGA-3' & 5'-TGAAGCCCGGGAATGAAGTC-3' \\
\hline Cyclophilin & 5'-TTGCTGCAGACATGGTCAAC-3' & 5'-TGTCTGCAAACAGCTCGAAG-3' \\
\hline
\end{tabular}

XO: Xanthine Oxidase; Cyt c ox: Cytochrome C Oxidase; SOD: Superoxide Dismutase; UCP: Uncoupling Protein.

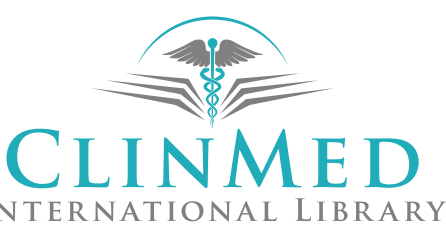

